precise nature of that evolution is ? I do hope, however, that we shall not be treated to another example of that confusion between evolution and mere development, which depends on a confusion of the inborn with the acquired and which is such a nuisance in so many popular treatises.

The letter of "M.D. Edin." is a little puzzling. If he means to imply, as I think he does, that heredity lies at the root of every social and moral question 1 agree with him. Hence the importance of the study. But if he means to imply, but of this I am very doubtful, that we should suppress the truth for fear of disturbing some of the arguments which clergymen have founded on error then I disagree very strongly. That would be profoundly immoral.

To Mr. Sidney Vines I need only reply that I have "no practical suggestion whatever to make concerning the prevention of drunkenness" at present. We are discussing quite a different question.

I am, Sirs, yours faithfully,

Southsea, Sept. 12th, 1903. G. ARChDALI Reid.

\section{OBSERVATIONS ON MASTICATION.}

\section{To the Editors of THE LANCET.}

SIRS,-Dr. Harry Campbell's objections to the use of antiseptic tooth powders and mouth washes have been assailed as being "quite contrary to belief, acknowledged practice, and observation." We know that they are contrary to belief and acknowledged practice, but to say that they are contrary to observation is wholly unjustifiable. Indeed, clinical evidence of the value of antiseptic mouth washes is conspicuous by its absence and the absolute failure of acknowledged practice to check the ravages of dental caries at the present day is one of the most dismal facts which we are compelled to recognise. I do not know that any observations or tabulated results have been recorded to show that those people who use such antiseptic methods are in any way more free from caries than others. On the other hand, the evi dence is overwhelming that peoples who consume food which compels efficient mastication are relatively free from dental troubles. In fact, dental caries is inversely proportional to the amount of mastication. I have particularly noticed that patients who carefully adopt the acknowledged practice are not only specially subject to "abrasion," "erosion," and recession of the gums, but are, as a rule, most liberally supplied with carious cavities or "fillings." I feel sure that the use of antiseptic mouth washes is only sanctioned by purely theoretical considerations. Dr. W. D. Millar showed that dental caries was a chemicoparasitical process and immediately he had done so he and the whole dental profession at once embraced the theory that we must destroy the micro-organisms and neutralise the acid in order to avoid the disease. It never was surmised that this chemico-parasitical process which causes the disintegration of the teeth was but an intensification or perversion of a normal process. Consider the nature of the micro-organisms of the mouth. Some are acid producers, some are liquefiers, and some produce acid or liquefy according to the medium in which they exist. It is obvious that those bacteria which liquefy gelatin and other albuminoid substances must be of great importance in rendering shreds of foodstuffs soluble and so helping to clean the buccal cavity, and within limits the acid producers may have a similar function. In fact it would seem impossible to do without the mouth bacteria as there is no ferment in the mouth capable of digesting the albuminous shreds and particles which otherwi-e would be bound to accumulate between and about the teeth. It may be said that at least we would be well to be without a considerable proportion of the acid-producing bacteria, for without acid it is impossible to have dental caries. No doubt this is so. But this result is best obtained by efficient mastication, for food which requires considerable mastication calls forth a corresponding flow of saliva which gets thoroughly incorporated with the food. The ptyaline converts the starch into a soluble form, while the mastication expresses it from the rest of the bolus. Thus, therefore, the starchy matter is more or less thoroughly swallowed, while the remaining part is being more completely masticated. By this means the smallest pos. sible residue tends to lodge about the teeth and what little does remain, being almost devoid of starch, allows the bacteria which liquefy to predominate while preventing the multiplication of the acid-producing bacteria. If, however, food-especially starchy food-is taken in a soft form the flow of saliva is, relatively to the amount of starch, but inefficiently stimulated. The starch is not fully converted and swallowed but remains plastered, as it were, into all the crevices in and about the teeth. Furthermore, the chronic lack of efficient stimulation of the salivary glands engendered by relatively soft foods leads, no doubt, to a corresponding lack of development and output of ptyaline. Now if there is a relatively great amount of starch left about the teeth can we wonder that the acid-producing bacteria largely preponderate and play havoc with the teeth?

of course, in exceptional circumstances, as during illness or after a very improper diet, the use of antiseptics may be advocated, "but under normal conditions their use is, I believe, to be deprecated."

I am, Sirs, yours faithfully,

Wimpole-street, W., Sept. 12th, $1903 . \quad$ J. SIM WALrACE.

\section{To the Editors of THF LANCET.}

SIRs, - The following experiment may be of interest during the controversy.

Eat a small piece of bread and notice the doughy feeling which it attains during the process. Brush the teeth with a solution of odol (I used the peppermint variety). Then eat another piece of bread and notice that the doughy feeling does not occur to anything like the same extent even after prolonged mastication. As a matter of fact, the gullet becomes a that the bread is still quite granular when it is swallowed. This would seem to indicate an arrest in the production of ptyaline and a failure in the conversion of the starch, but the solution of that question I leave to other hands. It is, at least, a result of the use of a powerfuliy antiseptic tooth cleanser. I am, Sirs, yours faithfully,

Guernsey, Sept. 14th, 1903.

J. AIKMAN.

\section{UMBILICAL CALCULUS AND THE URACHUS.}

To the Editors of THE LANGET.

SIRs, - The report published in THE LANCET of Sept. 12th, p. 747, of a Case of Umbilical Calculus by Mr. J. R. Harper and Dr. C. G. Seligmann is of considerable importance. It will interest those gentlemen to know that besides Shattock and Dean's cases about a dozen others have been collected by Ledderhose, Pernice, Volkmann, and Otto Hahn, whose article, "Ein Nabelkonkrement von Taubeneigrösse," was published three years ago in the Beiträge zur klinischen Chirurgie (vol. xxvi., p. 80). The umbilical calculus in Hahn's case measured 30 by 20 millimetres and was of the size and shape of a pigeon's egg. The patient, a man, aged 43 years, was of dirty habits-a common clinical feature, Hahn remarks, in all reported cases.

In relation to umbilical calculus is the question of urachal fistula, which I suggested in the article "Urachus" in the "Encyclopæedia Medica." Although the usual cause is no doubt irritation from accumulation of dirt it is possible that in some instances the upper portion of the canal of the urachus remains open, communicating by a minute orifice easily overlooked with the surface of the integument in the umbilicus. The patent canal would then be liable to infection, so that it would discharge morbid material into the umbilicus which would mix up with epidermis and foreign matter. Spencer Wells found urinary (?) concretions in the canal of a urachus which was closed at both ends and Wutz twice detected suppurative inflammation in a minute cystic dilatation representing an unobliterated portion of the urachal canal. Such conditions being known to exist we can understand how, if the uppermost part of the canal remained open, it might inflame and cause the development of concretions in the umbilicus.

I have repeatedly observed, when removing abdominal tumours, incomplete closure of the urachal canal and therefore I suspect that the urachus is more often a source of local mischief than is generally supposed. No doubt a wellestablished urachal fistula gives rise to very prominent symptoms and a vesical calculus in the dilated urachal canal, as in the case of Paget of Leicester, would never be mistaken for an umbilical calculus. Still, it is possible that in exceptional cases the urachal canal remains patent, though extremely narrow, throughout, so that when the bladder is unusually distended a few drops of urine might occasionally pass up it and irritate the integuments of the umbilicus. Tho 